Pacific

Journal of

Mathematics

\title{
IRREDUCIBLE NUMERICAL SEMIGROUPS
}

J.C. Rosales and M.B. Branco 


\title{
IRREDUCIBLE NUMERICAL SEMIGROUPS
}

\author{
J.C. Rosales and M.B. Branco
}

\begin{abstract}
We give a characterization for irreducible numerical semigroups. From this characterization we obtain that every irreducible numerical semigroup is either a symmetric or pseudosymmetric numerical semigroup. We study the minimal presentations of an irreducible numerical semigroup. Separately, we deal with the cases of maximal embedding dimension and multiplicity 3 and 4 .
\end{abstract}

\section{Introduction and basic concepts.}

A numerical semigroup is a subset $S$ of $\mathbb{N}$ closed under addition, $0 \in S$ and generates $\mathbb{Z}$ as a group (here $\mathbb{N}$ and $\mathbb{Z}$ denote the set of nonnegative integers and the set of the integers, respectively). From this definition we obtain (see $[\mathbf{2}]$ and $[\mathbf{1 3}]$ ) the following results.

(1) The set $\mathbb{N} \backslash S$ is finite, we refer to the greatest integer not belonging to $S$ as the Frobenius number of $S$ and denote it by $g(S)$.

(2) The semigroup $S$ has a unique minimal system of generators $\left\{n_{0}<\right.$ $\left.n_{1}<\cdots<n_{p}\right\}$. We refer to the numbers $n_{0}$ and $p+1$ as the multiplicity and embedding dimension of $S$ and denote them by $m(S)$ and $\mu(S)$, respectively.

Let $F=\left\{a_{0} X_{0}+\cdots+a_{p} X_{p}: a_{0}, \ldots, a_{p} \in \mathbb{N}\right\}$ be the free monoid generated by $\left\{X_{0}, \ldots, X_{p}\right\}$ and let $\varphi: F \rightarrow S$ be the monoid epimorphism defined by

$$
\varphi\left(a_{0} X_{0}+\cdots+a_{p} X_{p}\right)=a_{0} n_{0}+\cdots+a_{p} n_{p} .
$$

It is well-known that if $\sigma$ is the kernel congruence of $\varphi$ (that is, $x \sigma y$ if $\varphi(x)=\varphi(y)$ ), then $S$ is isomorphic to the quotient monoid $F / \sigma$ (see [13]). Rédei shows in [9] that the congruence $\sigma$ is finitely generated and therefore there exists

$$
\rho=\left\{\left(x_{1}, y_{1}\right), \ldots,\left(x_{t}, y_{t}\right)\right\} \subseteq F \times F
$$

such that $\sigma$ is the smallest congruence on $F$ that contains $\rho$. The set $\rho$ is called a presentation for the numerical semigroup $S$. We say that $\rho$ is minimal presentation if no proper subset of $\rho$ generates $\sigma$. In [10] it is shown that the concepts of minimal presentation and presentation with the lowest cardinality coincide for a numerical semigroup. 
Numerical semigroups have been widely studied in the literature not only from the semigroupist point of view but also to give us a series of examples in ring theory through the concept of the semigroup ring associated to a numerical semigroup (see for instance [7], [4], [5], [8], [15]). Along this line, if $K$ is a field, $K[S]$ is the finite type K-algebra associated to $S$ and $K\left[X_{0}, \ldots, X_{p}\right]$ is the polynomial ring in $p+1$ indeterminates, the $\mathrm{K}$-algebras epimorphism $\lambda: K[X] \rightarrow K[S]$ such that $X_{i} \mapsto t^{i}$ is a $S$-graded ring homomorphism with degree zero. Therefore, the prime ideal $P=\operatorname{kernel}(\lambda)$ (called the ideal associated to the semigroup) is homogeneous and defines a monomial curve in the $(p+1)$-dimensional affine space on $K$. Herzog proves in [7] that finding a system of generators for $P$ is equivalent to finding a presentation for $S$. Let us also notice that Kunz in [8] proves that $K[S]$ is Gorestein if and only if $S$ is symmetric and Barucci-Dobbs-Fontana prove in [2] that $K[S]$ is Kunz if and only if $S$ is pseudo-symmetric.

We say that a numerical semigroup is irreducible if it can not be expressed as an intersection of two numerical semigroups containing it properly. In Theorem 1, we see that $S$ is irreducible if and only if $S$ is maximal in the set of all numerical semigroups with Frobenius number $g(S)$. From [6] and [2] we deduce that the class of irreducible semigroups with odd (respectively even) Frobenius number is the same as the class of symmetric (respectively pseudo-symmetric) numerical semigroups. Moreover, [11] provides a study of the irreducible numerical semigroups with odd Frobenius number. Our aim in this paper is to generalize these results for irreducible numerical semigroups in general (that is, with Frobenius number even or odd).

The contents in this paper are organized as follows. In Section 2 we characterize the irreducible numerical semigroups giving special attention to their Apéry sets. In Section 3 we study the irreducible numerical semigroups with multiplicity 3 and 4 . We explicitly give the family of irreducible numerical semigroups of this kind. The aim of Section 4 is to give an upper bound for the cardinal of a minimal presentation for an irreducible numerical semigroup in function of their multiplicity and embedding dimension. Finally, in Section 5 we study those irreducible numerical semigroups having multiplicity greater than or equal to five and embedding dimension equal to its multiplicity minus one.

\section{Characterization of irreducible numerical semigroups.}

Throughout this section $S$ denotes a numerical semigroup, such that $S \neq \mathbb{N}$. It is well-known (see for instance [12]) that $S \cup\{g(S)\}$ is also a numerical semigroup.

Theorem 1. The following conditions are equivalent:

1) $S$ is irreducible, 
2) $S$ is maximal in the set of all numerical semigroups with Frobenius number $g(S)$,

3) $S$ is maximal in the set of all numerical semigroups that do not contain $g(S)$.

Proof. 1) $\Rightarrow 2$ ) Let $\bar{S}$ be a numerical semigroup such that $S \subseteq \bar{S}$ and $g(\bar{S})=$ $g(S)$. Then $S=(S \cup\{g(S)\}) \cap \bar{S}$. Since $S$ is irreducible, we deduce that $S=\bar{S}$.

2) $\Rightarrow 3)$ Let $\bar{S}$ be a numerical semigroup such that $S \subseteq \bar{S}$ and $g(S) \notin \bar{S}$. Then $\bar{S} \cup\{g(S)+1, g(S)+2, \ldots\}$ is a numerical semigroup that contains $S$ with Frobenius number $g(S)$. Therefore, $S=\bar{S} \cup\{g(S)+1, g(S)+2, \ldots\}$ and so $S=\bar{S}$.

$3) \Rightarrow 1)$ Let $S_{1}$ and $S_{2}$ be two numerical semigroups that contain $S$ properly. Then, by hypothesis, $g(S) \in S_{1}$ and $g(S) \in S_{2}$. Therefore $S \neq$ $S_{1} \cap S_{2}$ and so $S$ is irreducible.

From $[\mathbf{6}]$ and $[\mathbf{2}]$ we deduce the next result.

\section{Proposition 2.}

1) If $g(S)$ is odd, then $S$ is irreducible if and only if for all $h, h^{\prime} \in \mathbb{Z}$, such that $h+h^{\prime}=g(S)$, we have that either $h \in S$ or $h^{\prime} \in S$ (that is, $S$ is symmetric).

2) If $g(S)$ is even, then $S$ is irreducible if and only if for all $h, h^{\prime} \in$ $\mathbb{Z} \backslash\left\{\frac{g(S)}{2}\right\}$, such that $h+h^{\prime}=g(S)$, we have that either $h \in S$ or $h^{\prime} \in S$ (that is, $S$ is pseudo-symmetric).

Let $n \in S \backslash\{0\}$. Denote by $0=w(1)<w(2) \cdots<w(n)$ the smallest elements in $S$ in respective congruence classes $\bmod n$. We denote by $\operatorname{Ap}(S, n)$, the Apéry set of $n$ in $S$ (see [1]), the set $\{0=w(1)<w(2)<\cdots<w(n)\}$. It is well-known (see [13]) that $\operatorname{Ap}(S, n)=\{x \in S: x-n \notin S\}$ and that $w(n)=g(S)+n$.

The following result is also well-known (see [1], [4] or [11]):

Proposition 3. Let $n \in S \backslash\{0\}$. Then $S$ is irreducible with an odd Frobenius number (that is, $S$ is symmetric) if and only if $w(i)+w(n-i+1)=w(n)$ for all $i \in\{1, \ldots, n\}$.

Now we see how is the $\operatorname{Ap}(S, n)$ when $S$ is irreducible with an even Frobenius number.

Lemma 4. If $S$ is irreducible with an even Frobenius number and $n \in S \backslash$ $\{0\}$, then $\frac{g(S)}{2}+n \in \operatorname{Ap}(S, n)$.

Proof. It is enough to prove that $\frac{g(S)}{2}+n \in S$, since $\frac{g(S)}{2} \notin S$, but this follows from Proposition $2\left(\left(\frac{g(S)}{2}+n\right)+\left(\frac{g(S)}{2}-n\right)=g(S)\right)$. 
Proposition 5. Let $S$ be a numerical semigroup with an even Frobenius number and $n \in S \backslash\{0\}$. Then $S$ is irreducible if and only if

$$
\begin{aligned}
\operatorname{Ap}(S, n)=\{0=w(1)<w(2) & <\ldots \\
& <w(n-1)=g(S)+n\} \cup\{g(S) / 2+n\}
\end{aligned}
$$

and $w(i)+w(n-i)=w(n-1)$ for all $i \in\{1, \ldots, n-1\}$.

Proof. First note that if $g(S)$ is even, then $\frac{g(S)}{2}+n \in \operatorname{Ap}(S, n)$ and $\frac{g(S)}{2}+n<$ $\max \operatorname{Ap}(S, n)$. If $i \in\{1, \ldots, n-1\}$, then $w(i)-n \notin S$ and $w(i)-n \neq$ $\frac{g(S)}{2}$. By Proposition 2, we have that $g(S)-(w(i)-n) \in S$ and thus $w(n-1)-w(i)=g(S)+n-w(i) \in S$. Since $w(n-1) \in \operatorname{Ap}(S, n)$ we deduce that $w(n-1)-w(i) \in \operatorname{Ap}(S, n)$. Furthermore $w(n-1)-w(i) \neq \frac{g(S)}{2}+n$ because otherwise we would have $w(i)=\frac{g(S)}{2}$. Hence the reader can check that $w(i)+w(n-i)=w(n-1)$.

Conversely, let $x$ be an integer such that $x \neq \frac{g(S)}{2}$ and $x \notin S$. Let us show that $g(S)-x \in S$. Take $w \in \operatorname{Ap}(S, n)$ such that $w \equiv x(\bmod n)$. Then $x=w-k n$ for some $k \in \mathbb{N} \backslash\{0\}$. We distinguish two cases:

(1) If $w=\frac{g(S)}{2}+n$, then $g(S)-x=g(S)-\left(\frac{g(S)}{2}+n-k n\right)=\frac{g(S)}{2}+(k-1) n$. Besides, $x \neq \frac{g(S)}{2}$ leads to $k \neq 1$ and therefore $k \geq 2$. Hence we can assert that $g(S)-x \in S$.

(2) If $w \neq \frac{g(S)}{2}+n$, then $g(S)-x=g(S)-(w-k n)=g(S)+n-w+$ $(k-1) n=w(n-1)-w+(k-1) n \in S$, since $w(n-1)-w \in S$ by hypothesis.

Note that if $S$ has embedding dimension two, then $S$ is irreducible with odd Frobenius number (i.e., $S$ is symmetric); in fact $S$ is a complete intersection (see $[3,7]$ ).

Observe also that $\mu(S) \leq m(S)$ for every numerical semigroup $S$. The semigroups with $\mu(S)=m(S)$ have been widely studied in the literature (see for instance $[\mathbf{2}, \mathbf{1 2}, \mathbf{1 5}]$ ) and are called MED-semigroups (numerical semigroups with maximal embedding dimension).

Proposition 6. Let $S$ be an irreducible numerical semigroup.

1) If $g(S)$ is odd and $m(S) \geq 3$, then $\mu(S) \leq m(S)-1$.

2) If $g(S)$ is even and $m(S) \geq 4$, then $\mu(S) \leq m(S)-1$.

Proof. 1. See Section 2 of [11].

2. It is enough to prove that $\mu(S) \neq m(S)$. If $\mu(S)=m(S)$, then $S$ is minimally generated by $\left\{m(S), n_{1}, \ldots, n_{m(S)-1}\right\}$ and therefore $\operatorname{Ap}(S, n)$ is of the form

$$
\operatorname{Ap}(S, n)=\left\{0<n_{2}<\cdots<n_{m(S)-1}\right\} \cup\left\{n_{1}=\frac{g(S)}{2}+m(S)\right\} .
$$


Since $m(S)-1 \geq 3$ then $n_{1} \neq n_{2} \neq n_{m(S)-1}$. By Proposition 5 we deduce that $n_{m(S)-1}-n_{2} \in S$, which contradicts the fact that $\left\{m(S), n_{1}, \ldots\right.$, $\left.n_{m(S)-1}\right\}$ is a minimal system of generators for $S$.

Note that $S=\langle 3,7,11\rangle$ is an irreducible numerical semigroup with Frobenius number $g(S)=8$ (it is easy to see that 8 belongs to every numerical semigroup that properly contains $S$ ). That is why in 2) of the above proposition we need that $m(S) \geq 4$ instead of $m(S) \geq 3$.

Using 1) and 2) of the above proposition we can assert that if $S$ is an irreducible numerical semigroup with $m(S) \geq 4$, then $\mu(S) \leq m(S)-1$.

\section{Irreducible numerical semigroups with multiplicity 3 and 4 .}

In this section we study the irreducible numerical semigroups with multiplicity 3 and 4. By the remark made after Proposition 5, we know that if $\mu(S)=2$, then $S$ is irreducible. Recall also, that from Proposition 6 , if $m(S)=4$ and $S$ is irreducible then $\mu(S) \leq 3$.

Therefore, we focus our study in the cases:

1) $S$ is irreducible with $m(S)=\mu(S)=3$,

2) $S$ is irreducible with $m(S)=4$ and $\mu(S)=3$.

The following result is an immediate consequence of $[\mathbf{2}$, Theorems I.4.2, I.4.4]. Here we offer an alternative proof by using Apéry sets.

Theorem 7. The following conditions are equivalent:

1) $S$ is an irreducible numerical semigroup with $m(S)=\mu(S)=3$,

2) $S$ is generated by $\{3, x+3,2 x+3\}$ with $x$ not a multiple of 3 .

Proof. 1) $\Rightarrow 2$ ) If $m(S)=\mu(S)=3$, then $\left\{3, n_{1}, n_{2}\right\}$ is a minimal system of generators for $S$. From Proposition 6 we deduce that $g(S)$ is even and by Proposition 5 we have that

$$
\operatorname{Ap}(S, 3)=\left\{0, n_{1}=\frac{g(S)}{2}+3, n_{2}=g(S)+3\right\} .
$$

Taking $x=\frac{g(S)}{2}$ we have that $n_{1}=x+3$ and $n_{2}=2 x+3$. Furthermore, $x=\frac{g(S)}{2} \notin S$ and thus $x$ is not a multiple of 3 .

$2) \Rightarrow 1$ ) Clearly $\{3, x+3,2 x+3\}$ is a minimal system of generators for $S$ and thus $m(S)=\mu(S)=3$. We have that $\operatorname{Ap}(S, 3)=\{0, x+3,2 x+3\}$. Hence $2 x+3=g(S)+3$ and therefore $\frac{g(S)}{2}+3=x+3$. From Proposition 5 we deduce that $S$ is irreducible.

$S=\langle 3,3+x, 2 x+3\rangle$ is a MED-semigroup. Applying the results obtained in [12] we deduce that a minimal presentation for $S$ is:

$$
\rho=\left\{\left(2 X_{1}, X_{0}+X_{2}\right),\left(2 X_{2}, x X_{0}+X_{1}\right),\left((x+1) X_{0}, X_{1}+X_{2}\right)\right\} .
$$


Now we study the irreducible numerical semigroups with multiplicity 4 . We distinguish two cases taking into account that the Frobenius number is odd (a symmetric semigroup) or even (a pseudo-symmetric semigroup).

Herzog proves in [7] that a numerical semigroup $S$ with minimal system of generators $\left\{n_{0}, n_{1}, n_{2}\right\}$ is irreducible with an odd Frobenius number (i.e., symmetric) if and only if it is a complete intersection. Applying the results obtained in [5] this occurs if and only if $n_{i} \in\left\langle\frac{n_{j}}{\left(n_{j}, n_{k}\right)}, \frac{n_{k}}{\left(n_{j}, n_{k}\right)}\right\rangle$ for some $\{i, j, k\}=\{0,1,2\}$, where $\left(n_{j}, n_{k}\right)$ denotes the greatest common divisor (gcd for short) of $n_{j}, n_{k}$.

Theorem 8. The following conditions are equivalent:

1) $S$ is an irreducible numerical semigroup, $g(S)$ is odd, $m(S)=4$ and $\mu(S)=3$,

2) $S$ is a numerical semigroup generated by $\{4,2 x, x+2 y\}$ with $y \in \mathbb{N} \backslash\{0\}$ and $x$ an odd integer greater than or equal to 3 .

Proof. 1) $\Rightarrow 2$ ) If $m(S)=4$ and $\mu(S)=3$, then $\left\{4, n_{1}, n_{2}\right\}$ is a minimal system of generators for $S$. From the previous remark we only have two cases:

a) Assume that $d=\operatorname{gcd}\left\{4, n_{1}\right\}$ and $n_{2} \in\left\langle\frac{4}{d}, \frac{n_{1}}{d}\right\rangle$. Notice that $d=2$ and $n_{1}=2 x$ with $x$ an odd number greater than or equal to 3 . Furthermore $1=\operatorname{gcd}\left\{4, n_{1}, n_{2}\right\}$, then $n_{2}$ is an odd number and $n_{2} \in\langle 2, x\rangle$ thus $n_{2}=x+2 y$ (because all odd numbers in $\langle 2, x\rangle$ are of this kind).

b) Assume that $d=\operatorname{gcd}\left\{n_{1}, n_{2}\right\}$ and $4 \in\left\langle\frac{n_{1}}{d}, \frac{n_{2}}{d}\right\rangle$. From here we deduce that $n_{1}=2 d, n_{2}=k_{2} d$ with $k_{2}$ odd and $d$ an odd integer greater than or equal to 3 . Therefore, $n_{2}=d+\left(k_{2}-1\right) d$ with $\left(k_{2}-1\right) d$ even. Taking $x=d$ and $y=\frac{\left(k_{2}-1\right) d}{2}$ we obtain the desired result.

$2) \Rightarrow 1$ ) Clearly, $2=\operatorname{gcd}\{4,2 x\}$ and $x+2 y \in\left\langle\frac{4}{2}, \frac{2 x}{2}\right\rangle$. By the remark made before this theorem we have that $S$ is an irreducible numerical semigroup with an odd Frobenius number. Now, we need to show that $\{4,2 x, x+2 y\}$ is a minimal system of generators for $S$, but this is clear because:

1) $x+2 y \notin\langle 4,2 x\rangle$, since $x+2 y$ is odd,

2) $2 x \notin\langle 4, x+2 y\rangle$, since if $2 x=a 4+b(x+2 y)$ with $a, b \in \mathbb{N}$, then applying that $2 x$ is an even integer not a multiple of 4 and that $x+2 y$ is odd, we deduce that $b \geq 2$, contradicting that $2(x+2 y)>2 x$.

The semigroup $S=\langle 4,2 x, x+2 y\rangle$ has Frobenius number $g(S)=3 x+$ $2 y-4$, furthermore using that it is a complete intersection we deduce that a minimal presentation for $S$ is:

$$
\rho=\left\{\left(2 X_{1}, x X_{0}\right),\left(2 X_{2}, y X_{0}+X_{1}\right)\right\} .
$$


Finally, we study the irreducible numerical semigroups such that $g(S)$ is even, $m(S)=4$ and $\mu(S)=3$.

Theorem 9. The following conditions are equivalent:

1) $S$ is an irreducible numerical semigroup, $g(S)$ is even, $m(S)=4$ and $\mu(S)=3$,

2) $S$ is generated by $\{4, x+2, x+4\}$ with $x$ an odd integer greater than or equal to 3 .

Proof. 1) $\Rightarrow 2$ ) If $m(S)=4$ and $\mu(S)=3$, then $\left\{4, n_{1}, n_{2}\right\}$ is a minimal system of generators for $S$. From Lemma 4 we know that $\frac{g(S)}{2}+4 \in \operatorname{Ap}(S, 4)$. We distinguish two cases:

a) If $\frac{g(S)}{2}+4$ is a minimal generator then, by Proposition 5, we deduce that

$$
\operatorname{Ap}(S, 4)=\left\{0, n_{1}=\frac{g(S)}{2}+4, n_{2}, 2 n_{2}=g(S)+4\right\} .
$$

Taking $x=\frac{g(S)}{2}$, then $n_{1}=x+4$ and $n_{2}=x+2$. Furthermore $g(S) \notin S$ and therefore $x$ is odd.

b) If $\frac{g(S)}{2}+4$ is not a minimal generator, then

$$
\operatorname{Ap}(S, 4)=\left\{0, n_{1}, n_{2}, \frac{g(S)}{2}+4\right\} .
$$

Hence $g(S)+4=n_{1}$ or $g(S)+4=n_{2}$. Suppose that $g(S)+4=n_{1}$ then, by Proposition 5 , we deduce that $n_{1}-n_{2} \in S$, contradicting that $\left\{4, n_{1}, n_{2}\right\}$ is a minimal system of generators.

$2) \Rightarrow 1$ ) Clearly, $\{4, x+2, x+4\}$ is a minimal system of generators of $S$, whence $m(S)=4$ and $\mu(S)=3$. The reader can check that

$$
\operatorname{Ap}(S, 4)=\{0, x+2, x+4,2 x+4\} .
$$

Therefore $g(S)=2 x$ and then

$$
\operatorname{Ap}(S, 4)=\left\{0, \frac{g(S)}{2}+4, \frac{g(S)+4}{2}, g(S)+4\right\} .
$$

Using Proposition 5 we obtain that $S$ is irreducible.

Note that $S=\langle 4, x+2, x+4\rangle$ has Frobenius number $2 x$. Applying [7] and that this semigroup is not symmetric (therefore it is not a complete intersection), we can deduce that a minimal presentation for $S$ is:

$$
\rho=\left\{\left(2 X_{2}, X_{0}+2 X_{1}\right),\left(3 X_{1}, k X_{0}+X_{2}\right),\left(t X_{0}, X_{1}+X_{2}\right)\right\}
$$

with $k=\frac{3(x+2)-(x+4)}{4}$ and $t=\frac{(x+4)+(x+2)}{4}$. Observe that $3(x+2)-(x+4)$ is a multiple of 4 if and only if $x$ is odd, and $(x+4)+(x+2)$ is a multiple of 4 if and only if $x$ is odd. 


\section{An upper bound of the cardinality of a minimal presentation for an irreducible numerical semigroup.}

Let $S$ be a numerical semigroup with minimal system of generators $\left\{n_{0}<\right.$ $\left.n_{1}<\cdots<n_{p}\right\}$. In [12] it is shown the following result (\#MR $S$ denotes the cardinality of a minimal presentation for $S$ ).

Proposition 10. Let $S$ be a numerical semigroup. Then

$$
\# \mathrm{MR} S \leq \frac{n_{0}\left(n_{0}-1\right)}{2}-2\left(n_{0}-1-p\right) .
$$

In [11] this bound is improved in the case $S$ is symmetric. In fact, the following result is given there:

Proposition 11. If $S$ is symmetric, $n_{0} \geq 3$ and $p \geq 2$, then

$$
\# \mathrm{MR} S \leq \frac{\left(n_{0}-2\right)\left(n_{0}-1\right)}{2}-1+\left(p+2-n_{0}\right) .
$$

Our aim in this section is to prove the analogue to this result for $S$ an irreducible semigroup with even Frobenius number.

Throughout this section $S$ is an irreducible numerical semigroup with $g(S)$ even and $p \geq 3$.

For $n \in S$ define the graph $G_{n}=\left(V_{n}, E_{n}\right)$, as

$$
\begin{gathered}
V_{n}=\left\{n_{i} \in\left\{n_{0}, \ldots, n_{p}\right\}: n-n_{i} \in S\right\}, \\
E_{n}=\left\{\left[n_{i}, n_{j}\right]: n-\left(n_{i}+n_{j}\right) \in S, i, j \in\{0, \ldots, p\}, i \neq j\right\} .
\end{gathered}
$$

From $[\mathbf{1 2}]$ we can deduce the following result.

Proposition 12. If $\left\{n_{0}, n_{1}, \ldots, n_{p}, g(S)\right\}$ is a minimal system of generators for $S^{\prime}=S \cup\{g(S)\}, g(S)>n_{0}$ and $n_{i}$ and $n_{0}$ are in the same connected component of $G_{g(S)+n_{0}+n_{i}}$ for all $i \in\{1, \ldots, p\}$, then

$$
\# \mathrm{MR} S+p+2=\# \mathrm{MR} S^{\prime} \text {. }
$$

Applying Proposition 5 and using that $p \geq 3$ we deduce that $g(S)+n_{o} \geq$ $n_{i}+n_{j}$ for some $i, j \in\{1, \ldots, p\}$ and therefore $g(S)>n_{0}$. Furthermore, $\left\{n_{0}, n_{1}, \ldots, n_{p}, g(S)\right\}$ is a minimal system of generators for $S^{\prime}=S \cup\{g(S)\}$, since otherwise we would deduce from [12] that $n_{p}=g(S)+n_{0}$, which contradicts Proposition 5 for $p \geq 3$.

Lemma 13. If $i \in\{1, \ldots, p\}, w \in \operatorname{Ap}\left(S, n_{0}\right)$ and $n_{0}$ and $n_{i}$ are in two different connected components of $G_{w+n_{i}}$, then for all $w^{\prime} \in \operatorname{Ap}\left(S, n_{0}\right)$ such that $w-w^{\prime} \in S \backslash\{0\}$ we have that $w^{\prime}+n_{i} \in \operatorname{Ap}\left(S, n_{0}\right)$

Proof. Suppose that $w^{\prime}+n_{i} \notin \operatorname{Ap}\left(S, n_{0}\right)$, then $w^{\prime}+n_{i}-n_{0} \in S$. Let $s \in S \backslash\{0\}$ be such that $w=w^{\prime}+s$ and $j \in\{0, \ldots, p\}$ such that $s-n_{j} \in S$. Then, $w+n_{i}-\left(n_{i}+n_{j}\right) \in S$ and $w+n_{i}-\left(n_{j}+n_{0}\right) \in S$. Therefore $\left[n_{i}, n_{j}\right]$, $\left[n_{j}, n_{0}\right] \in E_{n}$ and so $n_{i}$ and $n_{0}$ are in the same connected component of $G_{w+n_{i}}$. 
Lemma 14. If $i \in\{1, \ldots, p\}$, then $n_{0}$ and $n_{i}$ are in the same connected component of $G_{g(S)+n_{0}+n_{i}}$.

Proof. Suppose that $n_{0}$ and $n_{i}$ are in two different connected components of $G_{g(S)+n_{0}+n_{i}}$. Let $j \in\{1, \ldots, p\}$ be such that $n_{j} \neq \frac{g(S)}{2}+n_{0}$ and $n_{i} \neq n_{j}$ (this is possible because $p \geq 3$ ). By Lemma 13 and Proposition 5 we deduce that $g(S)+n_{0}-n_{j}+n_{i} \in \operatorname{Ap}\left(S, n_{0}\right)$.

Observe that $g(S)+n_{0}-n_{j}+n_{i}=\frac{g(S)}{2}+n_{0}$, since otherwise using Proposition 5 we would obtain that $g(S)+n_{0}-\left(g(S)+n_{0}-n_{j}+n_{i}\right) \in S$ and therefore $n_{j}-n_{i} \in S$, contradicting that $\left\{n_{0}, \ldots, n_{p}\right\}$ is a minimal system of generators for $S$.

Let us observe that $n_{i} \neq \frac{g(S)}{2}+n_{0}$ because otherwise we would deduce from $g(S)+n_{0}-n_{j}+n_{i}=\frac{g(S)}{2}+n_{0}$, that $n_{j}=g(S)+n_{0}$ and applying Proposition 5 we can assert that $S=\left\langle n_{0}, \frac{g(S)}{2}+n_{0}, g(S)+n_{0}\right\rangle$, which contradicts that $p \geq 3$.

Now assume that $\operatorname{Ap}\left(S, n_{0}\right)=\left\{0=w(1)<\cdots<w\left(n_{0}-1\right)\right\} \cup\left\{\frac{g(S)}{2}+n_{0}\right\}$. We distinguish two cases:

1) If $\frac{g(S)}{2}+n_{0} \in\left\{n_{1}, \ldots, n_{p}\right\}$, then from Proposition 5 and Lemma 13 we have that

$$
w(1)+n_{i}=w(2), w(2)+n_{i}=w(3), \ldots, w\left(n_{0}-2\right)+n_{i}=w\left(n_{0}-1\right) .
$$

Hence,

$$
\operatorname{Ap}\left(S, n_{0}\right)=\left\{0, n_{i}, 2 n_{i}, \ldots,\left(n_{0}-2\right) n_{i}\right\} \cup\left\{\frac{g(S)}{2}+n_{0}\right\}
$$

and thus $S=\left\langle n_{0}, n_{i}, \frac{g(S)}{2}+n_{0}\right\rangle$, a contradiction because $p \geq 3$.

2) If $\frac{g(S)}{2}+n_{0} \notin\left\{n_{1}, \ldots, n_{p}\right\}$, then again from Proposition 5 and Lemma 13 we obtain that

$$
\begin{aligned}
\operatorname{Ap}\left(S, n_{0}\right)=\left\{0, n_{i}, \ldots, k n_{i}=\frac{g(S)}{2}+n_{0}, n_{j}, n_{j}\right. & +n_{i}, \ldots, \\
& \left.n_{j}+t n_{i}=g(S)+n_{0}\right\}
\end{aligned}
$$

for some $k, t \in \mathbb{N}$. Therefore, $S=\left\langle n_{0}, n_{i}, n_{j}\right\rangle$, in contradiction again with $p \geq 3$.

\section{Proposition 15.}

$$
\# \operatorname{MR} S \leq \frac{\left(n_{0}-2\right)\left(n_{0}-1\right)}{2}-1+\left(p+2-n_{0}\right) .
$$


Proof. Applying Lemma 14 and Proposition 12 we deduce that \#MRS = $\# \operatorname{MR}(S \cup\{g(S)\})-(p+2)$. From Proposition 10 we have that

$$
\# \operatorname{MR}(S \cup\{g(S)\}) \leq \frac{n_{0}\left(n_{0}-1\right)}{2}-2\left(n_{0}-1-p-1\right) .
$$

Hence,

$$
\# \mathrm{MR} S \leq \frac{\left(n_{0}-2\right)\left(n_{0}-1\right)}{2}-1+\left(p+2-n_{0}\right)
$$

From Propositions 15 and 11 we can obtain the following result.

Theorem 16. If $S$ is an irreducible numerical semigroup with $\mu(S) \geq 4$, then

$$
\# \mathrm{MR} S \leq \frac{(m(S)-2)(m(S)-1)}{2}-1+(\mu(S)+1-m(S)) .
$$

Note that if $\mu(S)=2$, then \#MR $S=1$ and if $\mu(S)=3$, then \#MR $S=2$ or 3 depending on the parity of $g(S)$ (see[7]).

\section{Irreducible numerical semigroups with maximal embedding dimension.}

A MEDI-semigroup is an irreducible semigroup with multiplicity $m \geq 5$ and embedding dimension $m-1$. Remember from Proposition 6 that if $S$ is irreducible and $m(S) \geq 5$, then $\mu(S) \leq m(S)-1$ and this is why we use the name MEDI-semigroup to indicate that it is an irreducible numerical semigroup with the maximal possible embedding dimension.

If $S=\left\langle m(S), n_{1}, \ldots, n_{m(S)-2}\right\rangle$ is a MEDI-semigroup, then

$$
\operatorname{Ap}(S, m(S))=\left\{0, n_{1}, \ldots, n_{m(S)-2}, g(S)+m(S)\right\} .
$$

Moreover, from Propositions 3 and 5 we can deduce that $g(S)+m(S)=$ $n_{i}+n_{j}$ with $i, j \in\{1, \ldots, m(S)-2\}$ and $i \neq j$. Applying now $[\mathbf{1 4}$, Theorem 1] we get that

$$
\# \mathrm{MR} S=\frac{(m(S)-2)(m(S)-1)}{2}-1 .
$$

Note that for $m(S) \in\{3,4\}$, the previous formula is not true (for this reason in the definition of MEDI-semigroup we need that $m(S) \geq 5$ ). In fact, for $m(S)=3$ applying the previous formula, we have \#MR $S=0$ but we know that a minimal presentation for $\left\langle 3, n_{1}\right\rangle$ has cardinal 1. For $m(S)=4$ applying the previous formula, we have \#MR $S=2$ and we know that in this class there are semigroups with minimal presentation of cardinal 3 (see the remark after Theorem 9 ).

If $S$ is a MEDI-semigroup with $g(S)$ odd, then $S$ is a MEDSY-semigroup according to the terminology used in [11]. 
Theorem 17. If $S$ is an irreducible numerical semigroup with $\mu(S) \geq 5$, then the following conditions are equivalent:

1) $S$ is a MEDI-semigroup, and

2) $\# \mathrm{MR} S=\frac{(m(S)-2)(m(S)-1)}{2}-1$.

Proof. 2) $\Rightarrow 1$ ) Since $\mu(S) \geq 4$, by Theorem 16 we know that

$$
\# \mathrm{MR} S \leq \frac{(m(S)-2)(m(S)-1)}{2}-1+(\mu(S)+1-m(S)) .
$$

Since

$$
\# \mathrm{MR} S=\frac{(m(S)-2)(m(S)-1)}{2}-1,
$$

we get that $\mu(S)=m(S)-1$ and therefore $S$ is a MEDI-semigroup.

$1) \Rightarrow 2$ ) Proved already (see the beginning of this section).

The next result appears in [11].

Lemma 18. Let $A=\{0=w(1), w(2), \ldots, w(m)\}$ be a subset of $\mathbb{N}$ such that $w(i) \not \equiv w(j)(\bmod m)$ for all $1 \leq i<j \leq m$, and let $S$ be a numerical semigroup generated by $A \cup\{m\}$. Then $\operatorname{Ap}(S, m)=A$ if and only if for all $1 \leq i, j \leq m$ there exist $1 \leq k \leq m$ and $t \in \mathbb{N}$ such that $w(i)+w(j)=$ $w(k)+t m$.

Proposition 19. If $S$ is an irreducible numerical semigroup with $m(S) \geq 5$ and

$$
\operatorname{Ap}(S, m(S))=\{0=w(1)<w(2)<\cdots<w(m(S))\},
$$

then the semigroup $S^{\prime}$ generated by

$$
\{m(S), w(2)+m(S), \ldots, w(m(S)-1)+m(S)\}
$$

is a MEDI-semigroup.

Proof. In [11, Proposition 2.4] it is proved that $\{m(S), w(2)+m(S), \ldots$, $w(m(S)-1)+m(S)\}$ is a minimal system of generators for $S^{\prime}$. Furthermore, in that proposition, it is also shown that if $S$ is symmetric, then $S^{\prime}$ is MEDSY-semigroup. Therefore it is enough to prove that if $S$ is irreducible with $g(S)$ even, then $S^{\prime}$ is irreducible. From Lemma 18 we obtain that

$$
\begin{array}{r}
\operatorname{Ap}\left(S^{\prime}, m(S)\right)=\{0<w(2)+m(S)<\cdots<w(m(S)-1)+m(S) \\
<w(m(S))+2 m(S)\}
\end{array}
$$

and, by Proposition 5, we get that $S^{\prime}$ is irreducible.

As a consequence of the previous proof we have that $g\left(S^{\prime}\right)=g(S)+2 m(S)$.

Proposition 20. If $S$ is a MEDI-semigroup with a minimal system of generators $\left\{m(S)<n_{1}<\cdots<n_{m(S)-2}\right\}$, then the semigroup $S^{\prime}$ generated by $\left\{m(S), n_{1}-m(S), \ldots, n_{m(S)-2}-m(S)\right\}$ is irreducible. 
Proof. In [11, Propositon 2.5] it is proved that if $S$ is a MEDSY-semigroup then $S^{\prime}$ is symmetric. Therefore, it is enough to prove that if $S$ is a MEDIsemigroup with $g(S)$ even, then $S^{\prime}$ is irreducible.

Assume that $n_{j}=\frac{g(S)}{2}+m(S)$ and

$$
\operatorname{Ap}(S, m(S))=\left\{0, n_{1}, \ldots, n_{m(S)-2}, g(S)+m(S)=n_{1}+n_{m(S)-2}\right\} .
$$

Using Lemma 18 it is easy to prove that

$$
\operatorname{Ap}\left(S^{\prime}, m(S)\right)=\left\{0, n_{1}-m(S), \ldots, n_{m(S)-2}-m(S), g(S)-m(S)\right\} .
$$

From Proposition 5 we conclude that $S^{\prime}$ is irreducible (note that $g\left(S^{\prime}\right)=$ $g(S)-2 m(S)$ and $\left.n_{j}-m(S)=\frac{g\left(S^{\prime}\right)}{2}+m(S)\right)$.

Applying Propositions 19 and 20 and a similar reasoning to the one used in the proof of $[\mathbf{1 1}$, Theorem 2.6] we obtain the following result:

Theorem 21. There is one to one correspondence between the irreducible semigroups with Frobenius number $g$ and multiplicity $m \geq 5$ and the MEDIsemigroups with Frobenius number $g+2 m$, multiplicity $m$ and the rest of minimal generators greater than $2 m$.

Acknowledgements. Special thanks to P. A. García-Sánchez and the referee for their comments and suggestions.

\section{References}

[1] R. Apéry, Sur les branches superlinéaires de courbes algébriques, C.R. Acad. Sci. Paris, 222 (1946), 1198-2000, MR 8,221a, Zbl 0061.35404.

[2] V. Barucci, D.E. Dobbs and M. Fontana, Maximality properties in numerical semigroups and applications to one-dimensional analytically irreducible local domains, Memoirs Amer. Math. Soc., 125(598) (1997), MR 97g:13039, Zbl 0868.13003.

[3] A. Brauer, On a problem of partitions, Amer. J. Math., 64 (1942), 299-312, MR 3,270d, Zbl 0061.06801.

[4] H. Bresinsky, On prime ideals with generic zero $x_{i}=t^{n i}$, Proc. Amer. Soc., 47 (1975), 329-332, MR 52 \#10741, Zbl 0296.13007.

[5] C. Delorme, Sous-monoides d'intersion complete de $\mathbb{N}$, Ann. Scient. Ec. Norm. Sup., 4-série, 9 (1976), 145-154, MR 53 \#10821, Zbl 0325.20065.

[6] R. Fröberg, G. Gottlieb and R. Häggkvist, On numerical semigroups, Semigroup Forum, 35 (1987), 63-83, MR 88d:20092, Zbl 0614.10046.

[7] J. Herzog, Generators and relations of abelian semigroups and semigroup rings, Manuscripta Math., 3 (1970), 175-193, MR 42 \#4657, Zbl 0211.33801.

[8] E. Kunz, The value semigroup of a one dimensional Gorenstein ring, Proc. Amer. Math. Soc., 25 (1970), 748-751, MR 42 \#263, Zbl 0197.31401.

[9] L. Rédei, The Theory of Finitely Generated Commutative Semigroups, Pergamon, Oxford-Edinburgh, New York, 1965, MR 32 \#5761, Zbl 0133.27904. 
[10] J.C. Rosales, An algorithm for determining a minimal relation associated to a numerical semigroup, Int. J. Algebra and Comput., 6 (1996), 441-455, MR 97f:20080, Zbl 0863.20026.

[11] _ On symmetric numerical semigroups, J. Algebra, 182 (1996), 422-434, MR 98h:20099, Zbl 0856.20043.

[12] _ On numerical semigroups, Semigroup Forum, 52 (1996), 307-318, MR 96m:20092, Zbl 0853.20041.

[13] J.C. Rosales and P.A. García-Sánchez, Finitely Generated Commutative Monoids, Nova Science Publishers, New York, 1999, MR 2000d:20074, Zbl 0966.20028.

[14] _ On numerical semigroups with high embedding dimension, J. Algebra, 203 (1998), 567-578, MR 99g:20108, Zbl 0906.20042.

[15] J. Sally, Cohen-Macaulay local rings of maximal embedding dimension, J. Algebra, 56 (1979), 168-183, MR 80e:14022, Zbl 0401.13016.

Received September 26, 2001 and revised February 26, 2002. The first author has been supported by the project BFM2000-1469. The second author has been supported by the University of Évora.

Departamento de Álgebra

UNIVERSIDAD DE GRANADA

E-18071 GRANADA

SPAIN

E-mail address: jrosales@ugr.es

Departamento de Matemática

UNIVERSIDADE DE ÉVORA

7000 ÉvOrA

Portugal

E-mail address: mbb@dmat.uevora.pt 